Canad. Math. Bull. Vol. 22 (4), 1979

\title{
A NECESSARY AND SUFFICIENT CONDITION FOR THE EQUIVALENCE OF THE TOPOLOGIES OF UNIFORM AND COMPACT CONVERGENCE
}

\author{
BY \\ MICHAEL H. CLAPP AND RAY C. SHIFLETT
}

1. Introduction. Let $(X, p)$ and $(Y, d)$ be metric spaces with at least two points. It is usual for introductory courses in topology to study the set $Y^{X}$ of all functions mapping $X$ to $Y$ with the pointwise, compact-open, uniform convergence, and uniform convergence on compacta topologies. Some care is taken to show sufficient conditions for these topologies to be equivalent $[1,2]$. However, the question of necessary conditions are dismissed with examples showing that the topologies are not in general equivalent. For instance, the topologies of uniform convergence on compacta and of uniform convergence are equivalent when $X$ is compact. The converse question seems to be unanswered. That is, if $X$ is not compact, are the topologies distinct? It is the purpose of this note to prove this converse theorem (Theorem 3, Section 3). To do so we introduce a new topology on $Y^{X}$ and show exactly when it lies properly between the topology of uniform convergence and the topology of uniform convergence on compacta (which we shall call compact convergence).

2. The topology of Cauchy convergence. The uniform convergence topology on the space of all functions $Y^{X}$ is induced by the metric

$$
u(f, g)=\sup \{\min [1, d(f(x), g(x))] \mid x \in X\} .
$$

The compact convergence topology has as a neighborhood base at $f \in Y^{X}$ the sets

$$
V(f, C, \varepsilon)=\left\{g \in Y^{X} \mid d(f(x), g(x))<\varepsilon \quad \text { for } \quad x \in C\right\}
$$

where $\varepsilon$ ranges over all positive reals and $C$ over all compact subsets of $X$.

Let $S$ be a sequence whose range is in $X$. The sets $V(f, S, \varepsilon), V(f, S, \varepsilon)=$ $\left\{g: d\left(f\left(x_{n}\right), g\left(x_{n}\right)\right)<\varepsilon\right.$ for all $n$ where $x_{n}$ is the $n$th term of $\left.S\right\}$ where $S$ is a Cauchy sequence, form a neighborhood base at $f \in Y^{X}$ for the topology of Cauchy convergence.

EXAMPLE 1. Let $\left(f_{n}\right)$ be a sequence of real-valued functions of a real variable defined by

$$
f_{n}(x)=\left\{\begin{array}{lll}
0 & \text { if } & x \leq n \\
1 & \text { if } & x>n
\end{array}\right.
$$

Received by the editors January 26, 1978 and, in revised form, November 27, 1978. 
The sequence converges uniformly to $f \equiv 0$ on every Cauchy sequence, that is, $\left(f_{n}\right)$ converges to $f \equiv 0$ in the topology of Cauchy convergence. However, $\left(f_{n}\right)$ does not converge in the topology of uniform convergence.

EXAMPLE 2. Let $\left(f_{n}\right)$ be the sequence defined on the open unit interval I by

$$
f_{n}(x)=x^{n}
$$

This sequence converges in the topology of compact convergence, that is, uniformly on all compact subsets of $I$. However, $\left(f_{n}\right)$ does not converge uniformly on any Cauchy sequence which converges to one. Thus, $\left(f_{n}\right)$ does not converge in the topology of Cauchy convergence.

Example 1 says that, in general, the topologies of uniform and Cauchy convergence are distinct. Example 2 says the topologies of Cauchy and compact convergence are distinct.

3. Results. In this section we present three theorems. The first two show the relationship between the three topologies discussed above and yield the third theorem which is our main result.

THEOREM 1. The topology of uniform convergence is finer than the topology of Cauchy convergence and they are equivalent if and only if $X$ is totally bounded.

Proof. The proof of the first part of the theorem is given by a Lemma.

LEMMA. The sequence $\left(f_{n}\right), f_{n} \in Y^{X}$, converges to $f$ in the topology of uniform convergence if and only if $\left(f_{n}\right)$ converges to $f$ uniformly on each countable subset of $X$.

Proof of the Lemma. Assume that $\left(f_{n}\right)$ does not converge in the topology of uniform convergence. Then there is an $\varepsilon>0$ such that for each positive integer $N$ one can find an $n \geq N$ and an $x_{N} \in X$ for which $d\left(f_{n}\left(x_{N}\right), f\left(x_{N}\right)\right) \geq \varepsilon$. This set $\left\{x_{N}\right\}$ is a countable set on which $\left(f_{n}\right)$ does not converge uniformly. Since the converse is obvious, the proof of the Lemma is complete. The Lemma says that the topology of uniform convergence is finer than the topology of Cauchy convergence, since the range of every Cauchy sequence in $X$ is a countable subset of $X$.

The proof of the equivalence of the topologies when $X$ is totally bounded is now reduced to showing that convergence of a sequence $\left(f_{n}\right)$ in the Cauchy convergence topology guarantees convergence in the topology of uniform convergence. Suppose not, then as in the Lemma, one can construct a countably infinite set $\left\{x_{n}\right\}$ in $X$ on which $\left(f_{n}\right)$ does not converge uniformly. Since $X$ is totally bounded, there is a subset of $\left\{x_{n}\right\}$ which is the range of a Cauchy sequence and on which $\left(f_{n}\right)$ does not converge uniformly, since, by the way in which $\left\{x_{n}\right\}$ was constructed, $\left(f_{n}\right)$ does not converge uniformly on any infinite countable subset of $\left\{x_{n}\right\}$. 
Finally, to complete the proof, we must show that when $X$ is not totally bounded the topologies are distinct. If $X$ is not totally bounded, then for some $\varepsilon>0$ there is a sequence $\left(x_{n}\right)$ in $X$ for which $p\left(x_{i}, x_{n}\right) \geq \varepsilon$ for all $i<n$.

Let $y_{1}$ and $y_{2}$ be distinct points in $Y$ and let

$$
f_{n}(x)=\left\{\begin{array}{lll}
y_{1} & \text { if } & x=x_{n} \\
y_{2} & \text { if } & x \neq x_{n}
\end{array}\right.
$$

Let $\left(z_{n}\right)$ by a Cauchy sequence in $X$. Then, for sufficiently large $N$, we have $p\left(z_{r}, z_{s}\right)<\varepsilon / 2$ when $r \geq N$ and $s \geq N$. Consequently, for $r \geq N$, we have $p\left(z_{N+1}, z_{\mathrm{r}}\right)<\varepsilon / 2$. Notice that at most one $x_{n}$ belongs to the sphere of radius $\varepsilon / 2$ about $z_{N+1}$ and at most the first $N$ of the $z_{n}$ lie outside this sphere. Therefore, at most $N+1$ of the $x_{n}$ and $z_{n}$ can be equal. It follows that $f_{m}\left(z_{r}\right)=y_{2}$ for all $z_{r}$ if $m$ is sufficiently large. If we take $f \equiv y_{2}$, we have the $\left(f_{n}\right)$ converges uniformly to $f$ on every Cauchy sequence. However, $\left(f_{n}\right)$ fails to converge uniformly to $f$ since $d\left(f_{n}\left(x_{n}\right), f\left(x_{n}\right)\right)=d\left(y_{1}, y_{2}\right)$ for all $n$. This concludes the proof of Theorem 1.

THEOREM 2. The topology of Cauchy convergence is finer than the topology of compact convergence and they are equivalent if and only if $X$ is complete.

Proof. Suppose $\left(f_{n}\right), f_{n} \in Y^{X}$, does not converge uniformly to $f$ on the compact set $C \subset X$. Then there exists a sequence in $C$ on which $\left(f_{n}\right)$ does not converge uniformly on any subsequence whose range is infinite. Since $X$ is a metric space, the sequence has a convergent subsequence and so the first part of the theorem is proven.

Now assume $X$ is complete and that a sequence $\left(f_{n}\right), f_{n} \in Y^{X}$, converges uniformly on compact sets. Given a Cauchy sequence $\left(x_{n}\right)$ in $X$ with the limit $x_{0},\left\{x_{n} \mid n=0,1, \ldots\right\}$ is compact. Thus $\left(f_{n}\right)$ converges uniformly on every Cauchy sequence in $X$.

Conversely suppose $X$ is not complete. Let $\left(x_{n}\right)$ by a Cauchy sequence which does not converge in $X$. Define $\left(f_{n}\right)$ by

$$
f_{n}(x)=\left\{\begin{array}{lll}
y_{1} & \text { if } & x=x_{n} \\
y_{2} & \text { if } & x \neq x_{n}
\end{array}\right.
$$

where $x \in X$ and $y_{1}$ and $y_{2}$ are distinct points of $Y$. If $C$ is a compact subset of $X$ then $C$ contains at most a finite number of the $x_{n}$, otherwise the sequence $\left(x_{n}\right)$ would converge in $X$. Therefore, $\left(f_{n}\right)$ converges uniformly to $f \equiv y_{2}$ on $C$. However, $\left(f_{n}\right)$ does not converge uniformly on $\left(x_{n}\right)$ since

$$
d\left(f_{n}\left(x_{n}\right), f\left(x_{n}\right)\right)=d\left(y_{1}, y_{2}\right)
$$

for all $n$. This concludes the proof of Theorem 2 .

As a direct consequence of Theorems 1 and 2 and the fact that compactness of a metric space is equivalent to completeness and total boundedness, we have our last and main result. 
THEOREM 3. The topology of uniform convergence on $Y^{X}$ is equivalent to the topology of compact convergence on $Y^{\mathrm{X}}$ if and only if $X$ is compact.

4. Remarks. While this result answers our initial question, there are several problems which apparently are still open and which we offer to stimulate some interest. For instance, if we restrict our attention to $C(X, Y$,$) , the set of$ continuous functions, are the theorems still valid? The proofs presented here do not hold. Do other topologies exist which fall between the uniform and compact convergence topologies? Do any exist between the pointwise topology and the compact-open or between the compact-open and the compact convergence? What is the situation when $X$ or $Y$ fail to be metric? (The authors wish to thank the referee for suggesting this last question and for helpful suggestions in the text.)

\section{BIBLIOGRAPHY}

1. James Dugundji, Topology, Allyn and Bacon, 1966.

2. William Pervin, Foundations of General Topology, Academic Press, 1964.

Department of Mathematics

California State Univ. Fullerton

Fullerton, CALIFORNIA 92634 